\title{
COMPARAÇÃO DA EFICÁCIA ENTRE EXERCÍCIOS EM CADEIA CINÉTICA ABERTA E FECHADA NO TRATAMENTO DE INDIVÍDUOS COM SÍNDROME FEMOROPATELAR
}

\author{
Paulo Henrique Silva Valentim dos SANTOS ${ }^{*}$, Newbson da Silva CÂNDIDO ${ }^{1}$ \& Michele Fontes SILVA ${ }^{2}$ \\ ${ }^{1}$ Faculdade da Cidade de Maceió - FACIMA. Maceió, Alagoas, Brasil. \\ ${ }^{2}$ Faculdade Estácio de Sá. Maceió, Alagoas, Brasil. \\ *Autor para correspondência: paulohvalentim@ hotmail.com \\ http://dx.doi.org/10.18571/acbm.081
}

\section{RESUMO}

A articulação do joelho envolve cerca de $50 \%$ das lesões musculoesqueléticas, sendo a síndrome femoropatelar (SDFP) a alteração mais comum, que corresponde a $20 \%$ dessa população. É caracterizada pela presença de dor difusa na região anterior e retropatelar do joelho, aumentando a dor ao realizar exercícios específicos. Os exercícios em cadeia cinética aberta (CCA) e cadeia cinética fechada $(\mathrm{CCF})$ aplicado no tratamento da SDFP promove redução da intensidade da dor, aumenta à força muscular, dentre outros. $\mathrm{O}$ objetivo deste foi comparar a eficácia entre o exercício em CCA e CCF em indivíduos com SDFP. Trata-se de um estudo de revisão de literatura analisando publicações dos últimos 11 anos nas bases de dados PubMed, SciELO e Medline. Foram incluídos no trabalho artigos que abordasse indivíduos com SDFP, exercícios em CCA e CCF, protocolos, ângulo Q, EMG, estudos entre 2003 a 2014. Foram excluídos os que não abordavam exercícios estudados em seu contexto. Quarenta e sete trabalhos foram pré-selecionados, 30 foram excluídos por não atenderem aos critérios de inclusão. Dessa forma, 17 estudos foram incluídos no trabalho tanto para confronto quanto para referencial teórico. Os estudos demonstraram que tanto os exercícios em CCA quanto os em CCF são eficazes no tratamento da SDFP, ambos mostraram resultados satisfatórios como melhora na capacidade funcional, redução da dor e diminuição do ângulo Q, contudo, os autores mostraram que os exercícios em CCF mostraram-se superior.

Palavras chave: Síndrome da dor femoropatelar, Terapia por exercícios, Reabilitação.

\begin{abstract}
The knee joint involves about $50 \%$ of musculoskeletal injuries, and the patellofemoral syndrome (PFPS) the most common change, which corresponds to $20 \%$ of this population. It is characterized by the presence of diffuse pain in the anterior region and retropatellar knee, increasing pain when performing specific exercises. The exercises in open kinetic chain (OKC) and closed kinetic chain $(\mathrm{CKC})$ applied for PPS treatment decreases the intensity of pain, increases muscle strength, among others. The objective of this was to compare the efficacy of exercise on OKC and CKC in individuals with PFPS. This is a literature review study analyzing the last 11 years publications in the databases PubMed, Medline and SciELO. Were included in the work items to approach individuals with PFPS, exercises in OKC and CKC, protocols, Q angle, EMG studies between 2003 and 2014. We excluded those that did not address exercises studied in context. Fortyseven works were pre-selected, 30 were excluded for not meeting the inclusion criteria. Thus, 17 studies were included in the study for both confrontation and to theoretical. Studies have shown that both the $\mathrm{OKC}$ exercises as the CKC are effective in treating PFPS, both showed satisfactory results
\end{abstract}


as improvement in functional capacity, reducing pain and decreasing the $\mathrm{Q}$ angle, however, the authors showed that the $\mathrm{CKC}$ exercises showed If higher.

Keywords: Patellofemoral Pain Syndrome, Exercise therapy, Rehabilitation.

\section{Introdução}

A articulação do joelho envolve cerca de $50 \%$ das lesões musculoesqueléticas, sendo a SDFP a alteração mais comum, que corresponde a $20 \%$ dessa população é muito comum em adolescentes e adultos jovens do sexo feminino, praticantes ou não praticantes de atividades físicas (ZANARDI, 2012; NOBRE, 2011; BARROSO, 2007; CABRAL, 2003).

Sua etiologia não se apresenta bem definida, sendo que os principais fatores são decorrentes do mau alinhamento patelar, podendo ser, um desequilíbrio de forças entre os músculos Vasto Medial Obliquo (VMO) e Vasto Lateral (VL), aumento do ângulo Q, rotação lateral da tíbia, patela alta ou baixa, anteversão femoral, pronaçãosubtalar excessiva, joelhos valgos ou varos, encurtamento do retináculo lateral ou dos músculos isquiotibiais (IQT) e trato iliotibial (MEIRA, 2012; CABRAL 2008; FEHR, 2006).

É caracterizada pela presença de dor difusa na região anterior e retropatelar do joelho, aumentando a dor ao realizar exercícios como subir ou descer escadas, ajoelhar, agachar, permanecer sentado por muito tempo e práticas de esportes. Ocorre devido ao aumento na força de compressão femoropatelar e também pode ser identificado através de sinais como crepitação patelar, edema, dor à palpação das bordas patelares e bloqueio articular (NOBRE, 2011; CABRAL, REMOTE, 2008; CABRAL, 2003).

O diagnóstico é realizado através de uma detalhada avaliação clínica que exige um domínio no conhecimento anatômico e biomecânico da articulação do joelho, associado a exames complementares como ressonância nuclear magnética, exames radiológicos ou tomografia computadorizada (MEIRA, ROQUE, 2012; CABRAL, 2008).

Inicialmente o tratamento conservador através da reabilitação é o mais indicado aos pacientes com SDFP, com finalidade de melhorar o quadro álgico, melhorar flexibilidade, aumentar a força e resistência dos músculos. Este tratamento consiste numa diversidade de técnicas, podendo citar os mais utilizados: alongamentos musculares, exercícios isotônicos, isométricos, proprioceptivos, CCA e CCF. Podendo ainda, associar aos recursos da eletroterapia e técnicas de taping de Grelsamer e McConnell (NAGASHIMA, ROQUE, ZANARDI, 2012; CABRAL, REMOTE, 2008; CABRAL, 2006).

Os exercícios em CCA e CCF nos últimos 15 anos tomou maior proporção dentro da reabilitação, inclusive vem sendo muito empregado no tratamento da SDFP. O exercício em CCA é caracterizado quando um membro se movimenta e a parte distal se encontra em movimento livre no espaço e apenas uma articulação está envolvida nesse movimento, onde se gera menos força compressiva com os joelhos em flexão de $90^{\circ}$ a $50^{\circ}$. Já os exercícios em CCF ocorrem quando em um movimento o seguimento distal se encontra fixo e envolve mais de uma articulação, esse movimento reduz a sobrecarga do joelho quando realizados entre $0^{\circ}$ e $50^{\circ}$ (MEIRA, ROQUE, SONTAG, ZANARDI, 2012; CABRAL, REMOTE, 2008).

Os exercícios em CCA e CCF aplicado no tratamento da SDFP promove redução da intensidade da dor, aumenta à força muscular, melhora a capacidade funcional, melhora a propriocepção e estabilidade articular e diminuição do estresse femoropatelar (NAGASHIMA, ZANARDI, 2012; BARROSO, 2007; CABRAL, 2006). 
O estudo teve como intuito comparar a eficácia entre os exercícios em CCA e CCF no tratamento da SDFP, descrevendo as alterações biomecânicas da mesma. Ainda, fundamentar cientificamente sobre a importância da abordagem fisioterapia neste grupo de indivíduo com o intuito de prevenir complicações inerentes a SDFP e possibilitar a recuperação funcional dos indivíduos.

\section{Materiais e Métodos}

\subsection{Identificação dos trabalhos}

Para identificar os trabalhos disponíveis na literatura sobre o tema em estudo, foram utilizadas as bases de dados da MEDLINE e LILACS por meio do PubMed (http://www.pubmed.gov) e SciELO (http://www.scielo.br).

Os seguintes descritores foram utilizados na busca: em língua inglesa, "patellofemoral pain syndrome", "exercise therapy", "rehabilitation" e, em língua portuguesa, "síndrome da dor femoropatelar", "terapia por exercícios", "reabilitação". Limitou-se a busca à presença dos descritores no título e/ou resumos dos artigos.

\subsection{Seleção dos estudos}

Foram selecionados os trabalhos que avaliaram e/ou compararam a efetividade de exercícios em CCA e CCF em indivíduos com SDFP. Buscaram-se, como variáveis: indivíduos com SDFP, exercícios em CCA e CCF, avaliação da dor de musculatura evolvida, protocolos, ângulo Q, EMG, estudos entre 2003 a 2014. Após a identificação dos descritores no título e/ou resumo, os artigos selecionados passaram por leitura dos resumos/abstracts para avaliar a adequação dos métodos e população de estudo, quanto à proposta desta revisão. Aqueles que apresentaram os critérios de elegibilidade predeterminados tiveram o texto completo adquirido para análise detalhada e extração dos dados. Foram excluídos os trabalhos que não abordavam os exercícios estudados em seu contexto e os que não se enquadravam do tempo determinado.

\subsection{Extração dos dados}

A busca e a análise dos artigos, de acordo com a estratégia supracitada, foram conduzidas de forma independente por dois avaliadores (Autor 1 e Autor 2) sem haver controvérsias quanto à elegibilidade de trabalhos para compor o banco de dados.

\subsection{Tabulação dos dados}

Os textos selecionados foram lidos detalhadamente para o levantamento dos dados correspondentes à SDFP e exercícios em CCA e CCF. Os dados relativos à caracterização da amostra estudada, variáveis estudadas e resultados obtidos nos estudos foram organizados em uma planilha do Microsoft Office Excel 2007. A partir daí, foi possível comparar a eficácia exercícios em CCA e CCF de indivíduos com SDFP. 


\section{Resultados}

Foram encontrados quarenta e sete artigos, sendo que, após o processo de seleção e análise dos mesmos, foram excluídos trinta estudos por não atenderem aos critérios de inclusão, sendo assim, utilizaram dezessete artigos para análise, confronto e comparação, bem como, para referência teórica através das introduções e conclusões dos mesmos.

Cinco trabalhos de intervenção foram encontrados. Apenas três estudos compararam exercícios em CCA e CCF, com divisão de dois grupos cada, onde cada grupo realizava exercícios específicos em CCA ou CCF e em ambos os estudos observou-se que há melhora na capacidade funcional e diminuição da dor, porém, fica evidente que os exercícios em CCF potencializam esses resultados por favorecer a ativação seletiva muscular (Tabela1).

Um estudo avaliou a intensidade da dor, capacidade funcional, ângulo Q e EMG dos músculos VM e VL, através de CCA, tendo diferenças estatisticamente significativas nessas variáveis quanto ao aumento da carga realizada no exercício. Outro avaliou a intensidade da dor ao realizar exercícios em CCF, dividindo dois grupos, indivíduos com e sem SDFP e constatou que a dor é maior induzida nos que apresentam SDFP em relação aos que não apresentam (Tabela 1). 
Tabela 1. Comparação de exercícios em CCA e CCF.

\begin{tabular}{|c|c|c|c|c|}
\hline AUTOR/ANO & ABORDAGEM & AMOSTRA & DESCRIÇÃO RESUMIDA & PRINCIPAIS RESULTADOS \\
\hline $\begin{array}{c}\text { Cabral et al. } \\
\qquad(2006)\end{array}$ & CCA & $\mathrm{N}=10$ & $\begin{array}{l}1 \text { Grupo: exercícios de fortalecimento do músculo QF na } \\
\text { cadeira extensora com aumento progressivo da carga, durante } \\
16 \text { sessões } 2 \text { vezes por semana. Sendo avaliados a intensidade } \\
\text { da dor, capacidade funcional, ângulo Q e EMG dos músculos } \\
\text { VM e VL. }\end{array}$ & $\begin{array}{l}\text { Os dados indicam diferença estatisticamente significante } \\
\text { para a variável dor, capacidade funcional e EMG do } \\
\text { músculo VL após o tratamento }(\mathrm{p}<0,05) \text {. }\end{array}$ \\
\hline $\begin{array}{c}\text { Fehr et al. } \\
\text { (2006) }\end{array}$ & $\mathrm{CCA}$ e $\mathrm{CCF}$ & $\mathrm{N}=24$ & $\begin{array}{l}2 \text { Grupos (SDFP): GI }(n=12) \text { realizou exercícios em CCA e } \\
\text { GII }(n=12) \text { realizou exercícios em CCF. Ambos os grupos } \\
\text { foram submetidos há } 8 \text { semanas consecutivas de tratamento } \\
\text { sendo } 3 \text { sessões semanais. Foram analisados os padrões de } \\
\text { ativação dos músculos VMO e VL através do EMG. }\end{array}$ & $\begin{array}{l}\text { Os grupos obtiveram redução significativa na intensidade da } \\
\text { dor e melhora no desempenho de atividades funcionais, } \\
\text { sendo que o GII mostrou-se superior ao GI nas duas } \\
\text { variáveis investigadas. Os resultados relativos à atividade } \\
\text { EMG mostraram que após o tratamento não houve } \\
\text { diferenças significativas na razão VMO/VL nas fases } \\
\text { excêntrica e concêntrica dos exercícios em CCA e CCF. }\end{array}$ \\
\hline $\begin{array}{l}\text { Merlin et al. } \\
\qquad(2006)\end{array}$ & CCA e CCF & $\mathrm{N}=20$ & $\begin{array}{l}2 \text { Grupos: G1 ( } n=10) \text { e } \mathrm{G} 2(\mathrm{n}=10) \text {, ambos realizaram } \\
\text { exercícios em CCA e CCF, respectivamente. Sendo } \\
\text { realizados } 2 \text { vezes por semana durante } 2 \text { meses, CCA } \\
\text { (cadeira extensora) e CCF (leg-press). }\end{array}$ & $\begin{array}{l}\text { Os exercícios em CCA e CCF apresentaram diminuição da } \\
\text { dor e do ângulo Q e melhora da capacidade funcional. } \\
\text { Ainda, os exercícios em CCF favorecem a ativação seletiva } \\
\text { do músculo VM em relação ao músculo VL. }\end{array}$ \\
\hline $\begin{array}{c}\text { Barroso et al. } \\
(2007)\end{array}$ & $\mathrm{CCF}$ & $\mathrm{N}=27$ & $\begin{array}{l}2 \text { Grupos: Grupo controle }(n=15) \text { e } \operatorname{SDFP}(n=12) \text {; Ambos } \\
\text { os grupos realizaram: Agachamento, step anterior e step } \\
\text { posterior, } 3 \text { repetições cada exercício com um ângulo de } \\
\text { flexão de joelho de } 45^{\circ} \text {. }\end{array}$ & $\begin{array}{l}\text { Os exercícios em CCF induziram maior intensidade de dor } \\
\text { nos sujeitos portadores de SDFP em relação aos sujeitos } \\
\text { sem a síndrome. }\end{array}$ \\
\hline $\begin{array}{c}\text { Cabral et al. } \\
\qquad(2008)\end{array}$ & $\mathrm{CCA}$ e $\mathrm{CCF}$ & $\mathrm{N}=20$ & $\begin{array}{l}2 \text { Grupos (SDFP): GI }(\mathrm{n}=10) \text { realizou fortalecimento do } \\
\text { músculo QF em CCA e GII }(n=10) \text { em CCF, ambos, durante } \\
8 \text { semanas com frequência de } 2 \text { vezes semanais. Foi avaliada } \\
\text { a dor, capacidade funcional, flexibilidade, encurtamento dos } \\
\text { músculos IQT, ângulo Q e EMG dos músculos VM e VL } \\
\text { durante extensão isométrica da perna. }\end{array}$ & $\begin{array}{l}\text { Houve melhora da capacidade funcional, encurtamento dos } \\
\text { músculos IQT e flexibilidade. Porém, somente o G1 } \\
\text { apresentou diminuição da intensidade da dor e aumento da } \\
\text { atividade EMG do músculo VL, enquanto ambos não } \\
\text { modificaram o ângulo Q. }\end{array}$ \\
\hline
\end{tabular}


Três estudos de revisão analisaram a comparação entre a eficácia dos exercícios em CCA e CCF em indivíduos com SDFP através de variáveis como: dor femoropatelar, melhora do ãngulo Q, ativação do músculo QF através da EMG e protocolos de intervenção. Pode-se observar que os mesmo chegam à conclusão de que ambos os exercícios em CCA e CCF são eficazes no tratamento de SDFP, mas que, quando aplicado CCF, este apresenta resultados potencialmente superiores aos em CCA por exercer maior sincronismo entre as musculaturas envolvidas, bem como o tratamento é mais eficaz quando este é aplicado através de protocolos específicos (Tabela 2).

Sendo assim, observa-se que ambas abordagens exercem papel fundamental na melhora funcional de indivíduos com SDFP, porém, quando aplicado CCF o resultado é potencializado.

Tabela 2. Comparação de exercícios em CCA e CCF em revisões de literatura.

\begin{tabular}{|c|c|c|c|c|}
\hline AUTOR/ANO & TEMPO & N. AUTORES & VARIAVEIS & PRINCIPAIS RESULTADOS \\
\hline $\begin{array}{l}\text { Neto et al. } \\
\text { (2011) }\end{array}$ & $2000-2010$ & $\mathrm{~N}=17$ & $\begin{array}{l}\text { Indivíduos com SDFP e que } \\
\text { tiveram como intervenção o } \\
\text { tratamento através da } \\
\text { cinesioterapia. }\end{array}$ & $\begin{array}{l}\text { Os exercícios em CCF foram os } \\
\text { mais indicados, devido ao fato } \\
\text { de exercerem maior sincronismo } \\
\text { entre o VMO, VLL e VLO e } \\
\text { menor estresse sobre a } \\
\text { articulação do joelho. }\end{array}$ \\
\hline $\begin{array}{l}\text { Nagashima et al. } \\
\text { (2012) }\end{array}$ & 2003-2012 & $\mathrm{N}=10$ & $\begin{array}{l}\text { SDFP e fisioterapia como } \\
\text { tratamento, bem como, } \\
\text { protocolos para } \\
\text { intervenção. }\end{array}$ & $\begin{array}{l}\text { Existe uma relação positiva } \\
\text { entre os protocolos de } \\
\text { tratamento para a SDFP, } \\
\text { principalmente no que se diz } \\
\text { respeito a exercícios em } \mathrm{CCF} \text {. }\end{array}$ \\
\hline $\begin{array}{l}\text { Sontag et al. } \\
\text { (2012) }\end{array}$ & $2005-2011$ & $\mathrm{~N}=11$ & $\begin{array}{l}\text { SDFP e exercícios em CCA } \\
\text { e CCF, melhor ângulo de } \\
\text { ativação do músculo } \\
\text { quadríceps através da EMG. }\end{array}$ & $\begin{array}{l}\text { Exercícios em CCF são } \\
\text { melhores, pois diminuem as } \\
\text { forças de cisalhamento e } \\
\text { enfatizam as co-contrações, } \\
\text { quando comparados aos de } \\
\text { CCA. No entanto, ambos os } \\
\text { exercícios reduzem a dor e } \\
\text { melhoram o desempenho nas } \\
\text { atividades funcionais em } \\
\text { indivíduos com SDFP. }\end{array}$ \\
\hline
\end{tabular}




\section{Discussão}

O joelho é uma das articulações que mais sofre lesões no corpo humano, devido à grande descarga de peso e aos movimentos estressantes, levando assim a diversos tipos de lesão, sendo a SDFP uma das alterações músculo-esqueléticas mais frequentes no joelho, correspondendo a aproximadamente 25\% dos diagnósticos ortopédicos (NETO, 2011; BEVILAQUA-GROSSI, 2005).

A SDFP é uma desordem dolorosa, onde o principal sintoma é uma dor na região anterior do joelho ou retropatelar, que é exacerbada ao realizar atividades como subir e descer escadas, agachar, correr e permanecer sentado por um longo período. Acomete adultos jovens do sexo feminino e atletas de ambos os sexos. Apesar de não apresentar etiologia bem definida, pode estar relacionada a alterações biomecânicas (SONTAG, 2012; MOREIRA, 2009; PULZATTO, 2005).

Inicialmente o tratamento conservador é o mais indicado, que tem como objetivo promover um equilíbrio entre as porções do músculo quadríceps, através dos exercícios em CCA e CCF. Nos exercícios de CCA a parte distal da extremidade não está fixo, e sim, livre no espaço, permitindo que o seguimento se movimente de forma isolada. Já nos exercícios de CCF a parte distal está fixa, esse movimento envolve mais de uma articulação. Esses exercícios geram a contração simultânea dos músculos agonistas e antagonistas, que proporciona grande estabilização articular (SONTAG, 2012; NETO, NOBRE, 2011).

Cabral (2008) realizou um estudo onde participaram 21 mulheres com SFP, divididos em dois grupos: Grupo 1 (G1) com 11 mulheres realizou fortalecimento do músculo Quadríceps Femoral (QF) em CCA na cadeira extensora e Grupo 2 (G2) com 10 mulheres realizou fortalecimento do músculo QF em CCF no leg-press. Foram avaliadas intensidade da dor, índices das escalas de capacidade funcional, flexibilidade e encurtamento dos músculos IQT, ângulo Q e Eletromiografia (EMG) dos músculos VM e VL durante extensão isométrica da perna.

Após o tratamento, os resultados mostram que houve melhora da capacidade funcional em ambos os grupos. Entretanto, somente o G1 apresentou diminuição da intensidade da dor e aumento da atividade EMG do músculo VL, nenhum grupo mostrou mudanças no ângulo Q. Os dados da pesquisa sugerem que os tratamentos baseados no fortalecimento do músculo QF oferecem melhoras importantes nos principais sinais e sintomas apresentados pelas pacientes, não havendo diferenças evidentes entre os realizados em CCA e CCF (CABRAL, 2008).

Já Fehr (2006) realizou um estudo com 24 voluntários portadores de SDFP, foram aleatoriamente divididos em dois grupos: grupo I $(n=12)$ : realizou exercícios em CCA na mesa flexo-extensora (90 a 50 graus); grupo II $(n=12)$ : realizou exercícios em CCF de semi-agachamento (0 a 50 graus) e exercícios isométricos de QF realizados no aparelho de pressão das pernas a 45 graus. $\mathrm{O}$ tratamento consistiu em 3 sessões semanais durante 8 semanas em dias alternados.

Foram analisados os padrões de ativação dos músculos VMO e VL através dos sinais F 8 j, também foram avaliados a intensidade da dor e funcionalidade dos voluntários por meio da Escäıa Visual Analógica (EVA) e escala de Kujala. De acordo com as condições experimentais utilizadas as análises dos valores de ativação do VMO e VL não mostrou diferença significativa nos dois grupos, ainda assim, o músculo VMO apresentou menor taxa de ativação em relação ao VL na fase excêntrica do exercício em CCF. Foram encontrados aumentos significativos na funcionalidade e redução da intensidade da dor em ambos os grupos, porém, o grupo II mostrou-se superior ao grupo I nestas duas variáveis. Diante dos achados analisados nesse estudo os exercícios em CCF mostraram-se mais eficazes comparados com os em CCA (FEHR, 2006).

Os estudos realizados por Cabral (2008) e Fehr (2006) são semelhantes, porém, Cabral afirma que os exercícios em CCA e CCF mostram-se eficaz no fortalecimento do músculo QF, onde 
não há diferenças evidentes entre os mesmos. Já no estudo de Fehr, os dois exercícios mostraram resultados satisfatórios, sendo que os exercícios em CCF se mostraram superior em relação ao CCA.

O estudo realizado por Melin (2006) contou com a participação de 20 mulheres, sedentárias, na faixa etária de 18 a 30 anos, foram distribuídas de forma aleatória em 2 grupos: grupo 1 (G1) e grupo 2 (G2), ambos realizaram exercícios em CCA e CCF. Os exercícios em CCA foram realizados na cadeira extensora e os em CCF no Leg-Press em duas sessões semanais, durante 2 meses. Também foram avaliadas a intensidade da dor, ângulo Q, capacidade funcional e EMG dos músculos VM e VL.

Os resultados obtidos nesse estudo indicam que os exercícios em CCA e CCF, quando realizados com controle da Amplitude de Movimento (ADM) e com monitorização da intensidade da dor, podem ser eficientes no tratamento de pacientes com SDFP, uma vez que houve diminuição do ângulo Q, aumento da atividade elétrica do músculo VM, diminuição da intensidade da dor e melhora da capacidade funcional em ambos (MERLIN, 2006).

Porém, devemos citar que, embora tenham obtido aumento da atividade do músculo VM em ambos os grupos, este foi mais notável no G1 do que no G2, contudo, no G1 houve também um aumento de maior proporção na atividade do músculo VL quando em comparação com o músculo $\mathrm{VM}$, enquanto que no G2, houve aumento na atividade do músculo VM e diminuição na ativ ${ }^{*}$ le do músculo VL, o que pode ser mais esperado para o tratamento da SDFP (MERLIN, 2006).

De acordo com Melin (2006), tanto os exercícios em CCA e os CCF mostraram eficácia no tratamento da SDFP, desde que sejam realizados em amplitudes controladas, ambos mostraram bons resultados, contudo, os exercícios em CCF mostrou ser mais efetivo no tratamento da SDFP.

Barroso (2007) realizou uma pesquisa com exercícios em CCF onde participaram 12 mulheres com SDFP (21,08 \pm 2,31 anos) e 15 mulheres saudáveis e sedentárias $(21,13 \pm 2,17$ anos), onde realizaram 3 atividades: agachamento isométrico com joelhos flexionados a $45^{\circ}$ e descida anterior e posterior de um step com altura regulável de modo que o joelho atingisse no máximo $45^{\circ}$ de flexão no momento do exercício. Após 3 repetições de cada exercício as voluntárias foram submetidas ao preenchimento da EVA.

A análise dos dados mostrou que a intensidade da dor dos indivíduos do grupo com SDFP foi significativamente maior quando comparada à dor registrada no grupo controle durante a realização dos exercícios de agachamento e dos exercícios de descida posterior e anterior do Step. Estes exercícios podem ser aplicados na avaliação funcional, contudo, devem ser utilizados com cuidado na reabilitação, sendo mais indicados nas fases finais. Afirma ainda, que os exercícios em CCF geram um aumento da intensidade da dor ao realizar os exercícios, assim, é mais segura a sua prática nas fases intermediária e final (BARROSO, 2007).

Em um ensaio clinico realizado por Cabral (2006), 11 pacientes do sexo feminino com SDFP, sedentárias, com idade entre 18 e 32 anos. Realizaram fortalecimento do músculo QF em CCA na cadeira extensora 2 vezes por semana no total de 16 sessões. Foram avaliadas as seguintes variáveis: intensidade da dor, capacidade funcional, ângulo Q e EMG dos músculos VM e VL.

Nos resultados obtidos antes e após o tratamento todas as variáveis apresentaram diferença estatisticamente significante após o tratamento, exceto o ângulo Q e a atividade EMG do músculo VM. Este estudo sugere que os exercícios de fortalecimento do músculo QF com controle da ADM devem ser incluídos no tratamento de pacientes com SDFP, já que produzem resultados funcionais satisfatórios, consideravelmente na dor e capacidade funcional (CABRAL, 2006). De acordo com o mesmo, o exercício em CCA mostrou-se efetivo no tratamento da SDFP, na redução da intensidade da dor e melhora da capacidade funcional. 
Sontag (2012) em seu estudo realizou uma seleção de artigos científicos com o intuito revisar e comparar os exercícios em CCA com os em CCF, a fim de verificar qual é mais eficiente e quais os ângulos que melhor ativam o músculo QF nesses exercícios por meio da EMG, para recuperação funcional dos portadores da SDFP. Com base no estudo realizado concluiu-se que ambos os exercícios reduzem a dor e melhoram o desempenho nas atividades funcionais, quando realizados em angulações seguras, sendo que os exercícios em CCF se mostraram melhores, pois diminuem as forças de cisalhamento e enfatizam as co-contrações, quando comparados aos de CCA.

Neto (2011) realizou um estudo do tipo revisão, onde analisou os benefícios da cinesioterapia, buscando identificar a melhor forma de aplicação desse tratamento na SDFP. Os resultados desse estudo evidenciaram que o tratamento através da cinesioterapia mostrou- se eficaz, sendo os exercícios em CCF os mais indicados, pois exercem maior sincronismo entre os músculos VMO, VLL e VLO e menor estresse na articulação do joelho.

No estudo de Nagashima (2012) foi realizado uma revisão de literatura, onde ele avaliou como os autores pesquisaram os efeitos da fisioterapia no tratamento SDFP. Ao final de sua pesquisa o mesmo concluiu que existe uma relação positiva entre os protocolos de tratamento para a disfunção femoropatelar, principalmente no que se diz respeito a exercícios em cadeia cinética fechada.

\section{Conclusão}

Contudo, os estudos demonstraram que tanto os exercícios em CCA quanto os em CCF são eficazes no tratamento da SDFP, ambos mostraram resultados satisfatórios como melhora na capacidade funcional e redução da dor, contudo, os autores mostraram que os exercícios em CCF mostraram-se superior por atingir a ativação seletiva muscular o que traz melhores resultados através do EMG e capacidade funcional. Novas pesquisas devem ser realizadas, para o melhor esclarecimento sobre o assunto.

\section{Referências}

Barroso VV et al. Quantitative pain analysis during closed kinetic chain exercises in subject: 11 patellofemoral pain syndrome. Fisioterapia Especialidades - Volume 1 - Número 1 - outubro / dezembro de 2007.

Bevilaqua-Grossi D et al. Avaliação eletromiográfica dos músculos estabilizadores da patela durante exercício isométrico de agachamento em indivíduos com síndrome da dor femoropatelar. RevBrasMed Esporte _ Vol. 11, Nº 3 - Mai/Jun, 2005.

Cabral CMN et al. Tratamento fisioterapêutico da síndrome fêmoropatelar com exercícios em cadeia cinética aberta. USP/SP, (2006).

Cabral CMN, Monteiro-Pedro V.Recuperação funcional de indivíduos com disfunção fêmoropatelarpor meio de exercícios em cadeia cinética fechada: revisão da. Rev. bras. fisioter. Vol. 7, No. 1 (2003), 1-8.

Cabral CMN. Physical therapy in patellofemoralsyndrome patients:comparison of open and closed kinetic chain exercises. ActaOrtop Bras 16(3:180-185, 2008). 
Fehr GL et al. Efetividade dos exercícios em cadeia cinética aberta e cadeia cinética fechada no tratamento da síndrome da dor femoropatelar. RevBrasMed Esporte _ Vol. 12, No 2 - Mar/Abr, 2006.

Meira SS et al. Síndrome da dor femoropatelar: Uma revisão. Brazilian Journal of Biomechanics, Year 2012, vol 13, n.25.

Melim AMO, Cabral CMNC. Análise comparativa de exercícios em cadeia cinética aberta e fechada no tratamento de pacientes com disfunção fêmoro-patelar. Revista PIBIC, Osasco, v. 3, n. 1, 2006, p. $45-53$.

Moreira LS et al. Comparação dos exercícios step e levantar/sentar na reabilitação da síndrome dolorosa femoropatelar: análise eletromiográfica dos músculos vastos medial e lateral. Universidade Federal de Goiás, 2009.

Nagashima MLB, Inoue MMEA. Patellofemoral dysfunction treatment protocols. Revista Eletrônica Saúde: Pesquisa e Reflexões - Volume 2- nº $1-2012$.

Neto MG et al. Aplicação e efeitos da cinesioterapia em indivíduos com síndrome da dor femoropatelar. Revista Científica da UNIRB - Ano III - Junho/2011.

Nobre TL.Comparison of exercise open kinetic chain and closed kinetic chain in the rehabilitation of patellofemoral dysfunction. Fisioter Mov. 2011 jan/mar; 24(1): 167-72.

Pulzatto F et al. Stepheight influence on backward step-up exercise: An electromyographic study in healthy individuals and in those withpatellofemoral pain syndrome. ActaOrtop Bras 13(4) - 2005.

Remonte Junio, JJHanf AG. Abordagem fisioterapêutica na síndrome da dor Fêmoropatelar: Revisão bibliográfica. Associação Cultural Educacional de Itapeva Faculdade de Ciências Sociais e Agrárias de Itapeva, SP, 2008.

RoqueV et al. PatellofemoralSyndrome. Revista da Sociedade Portuguesa de Medicina Física e de Reabilitação. Vol 22 I No 2 I Ano 20 (2012).

Sontag AA et al. Síndrome da dor femoropatelar: exercícios em cadeia cinética aberta e fechada. Breve revisão. EFDeportes.com, Revista Digital. Buenos Aires - Ano 17 - No 168 - Maio de 2012.

Zanardi CC, Lima MCAM.Intervenção fisioterapêutica em pacientes portadores da síndrome femoropatelar.Saúde Meio Ambient. v. 1, bn. 1, jun. 2012. 